\title{
1 Variability in amylose content of Bangladeshi rice cultivars due to uniqueSNPs in Waxy allele
}

2 Saima Shahid ${ }^{\mathrm{a}, \mathrm{c}, 1}$, Rokeya Begum $^{\mathrm{a}, \mathrm{c}}$, Samsad Razzaque ${ }^{\mathrm{a}}$, Jesmin $^{\mathrm{b}}$ and Zeba I. Seraj ${ }^{\mathrm{a}^{*}}$

3 aPlant Biotechnology Laboratory, Department of Biochemistry and Molecular Biology, University of

4 Dhaka, Dhaka-1000, Bangladesh

$5{ }^{\mathrm{b}}$ Department of Genetic Engineering and Biotechnology, University of Dhaka, Dhaka-1000,

6 Bangladesh

7

$8 \quad{ }^{\mathrm{c}}$ Contributed equally

10 *Corresponding Author: Zeba I. Seraj, Plant Biotechnology Laboratory, Department of Biochemistry

11 and Molecular Biology, University of Dhaka, Dhaka-1000, Bangladesh. Tel: +880-2-8614708; fax:

12 +880-2-8615583/9127051. Email: zebai@du.ac.bd.

13

$14{ }^{1}$ Present address: Plant biology PhD program, Department of Biology, and the Huck Institutes of the

15 Life Sciences, Penn State University, University Park, PA 16802, USA

16 


\section{List of Abbreviations}

$18 \quad \mathrm{AC}=$ Amylose content

$19 \mathrm{bp}=$ Base pairs

20 cDNA $=$ Complementary DNA

$21 \mathrm{CTAB}=$ Cetyltrimethylammonium bromide

22 DNA =Deoxyribonucleic acid

23 dNTP = Deoxynucleoside triphosphate

$24 \quad \mathrm{ESE}=$ Exonic splicing enhancer

25 GBSS-I = Granule-bound starch synthase I

26 G3PDH = Glyceraldehyde-3-phosphate dehydrogenase

27 indel $=$ Insertion/deletion

$28 \mathrm{~kb}=$ Kilobase pairs

$29 \mathrm{mM}=$ Millimolar

30 mRNA $=$ Messenger RNA

31 ng = Nanogram

$32 \mathrm{pmol}=$ Picomole

33 QTL $=$ Quantitative trait loci

$34 \quad \mathrm{RNA}=$ Ribonucleic acid

35 RT-PCR $=$ Reverse transcription polymerase chain reaction

$36 \mathrm{SF} 2 / \mathrm{ASF}=$ Splicing factor-2/ alternative splicing factor

37 SNP $=$ Single nucleotide polymorphism

$38 \quad \mathrm{UTR}=\mathrm{Untranslated}$ region

$39 W x=W a x y$

$40 \mu \mathrm{l}=$ Microlitre

$41 \mu \mathrm{M}=$ Micromolar 


\section{Abstract}

48 Waxy gene (Granule Bound Starch Synthase I) is responsible for amylose synthesis in the rice 49 endosperm.Several mutations in this gene have been shown to be responsible for variable amylose 50 content (AC) phenotypes. The G/T mutation in $5^{\prime}$ splice site of Waxy intron 1 has been traced as the 51 origin of the glutinous rice phenotype and differentiates low AC fromnon-glutinous intermediate/high 52 AC rice. Sequencing of Waxypromoter and 5' noncoding regions from 22 rice cultivars showed that 53 the evolutionary pattern of all Bangladeshi non-glutinous and most glutinous rice accessions are in 54 line with the general patternsof South and Southeast Asia. However, three cultivars Khara Beruin, 55 Modhu Beruin White and Kathali Beruin Red with low to very low amylose lackedthe G/T splice site 56 mutation. These were more closely related to non-glutinous cultivars based on their SNP patterns in 57 promoter and noncoding regions. Further sequencing revealed a unique $\mathrm{C}$ deletion at a pyrimidine 58 tract of intron 5 of these three cultivars that may cause slippage of intron splicing. Additional SNPs at 59 intron 9 and 10 were also identified among these cultivars. These Bangladeshi-genotype-specific 60 mutations could be the cause of waxy or low amylose phenotypes in theseglutinous accessions.

62 Key words: Amylose content; Waxy gene; Glutinous and non-glutinous rice; SNPs 


\section{Introduction}

64 Starch in rice endosperm contains amylose and amylopectin polysaccharides. Amylose is principally a 65 linear molecule containing $\alpha(1 \rightarrow 4)$ linked glucose units and makes up approximately $0-30 \%$ of total starch.In contrast amylopectin is a branched molecule which contributes to approximately $70-100 \%$ of total starch in rice endosperm (Martin and Smith, 1995). Higher amylose levels (20-30\%) are observed in many indica rice varieties in South Asia (Morishima et al., 1992). Lower amylose levels $(10-20 \%)$ are more common in japonica varieties that predominate in East Asia (Yamanaka et al., 2004). Amylose content (AC) can be thus used to classify milled rice samples into different categories such as glutinous/waxy (0-5\% amylose), low AC (6-18\%), intermediate AC (19-23\%), or high AC $(>23 \%)$ types(Bergman et al., 2004). Low AC is usually associated with tender, cohesive, and glossy cooked rice while high AC is associated with firm, fluffy, and separated grains incooked rice(Juliano et al., 1981). Therefore, AC is considered asone of the major characteristics for assessingrice cooking and eating qualities. In addition, low $\mathrm{AC}$ rice varieties have long been used as a tool of improving grain quality through conventional breeding as theserepresent an intermediate type between glutinous and non-glutinous rice varieties (Dong, 2000; Sato et al., 2002).

Genetic and molecular marker-based QTL analyses have revealed that the wide range of AC variationin rice endosperm is mainly controlled by a major locus (Wx or Waxy gene) and multiple minor loci(Fan et al., 2005; Inukai et al., 2000). The Wxgene in rice encodes the granule-bound starch synthase I (GBSS-I) enzymeresponsible for amylose biosynthesis (Okagaki and Wessler, 1988).The origin of the glutinous phenotype such as those found in japonica rice has been traced to the $W x^{b}$ allele, which contains a G/T mutation in intron 1 splice donor site of the $W x$ gene(Olsen and Purugganan, 2002). Presence of this splice donor site mutation leads to inefficient splicing of $W x$ premRNA followed by reduced expression of functional GBSS-I (Cai et al., 1998). In contrast, nonglutinousphenotype observed in many indica rice varieties has been traditionally associated with $W x^{a}$ allele, which lacks the intron $1 \mathrm{G} / \mathrm{T}$ mutation. Howevermany Northeast Asian rice varieties carrying thisintron $1 \mathrm{G} / \mathrm{T}$ mutation still show non-glutinous phenotype. This suggests that partial suppression of the intron $1 \mathrm{G} / \mathrm{T}$ mutation may have an important role in the development of non- 
91 glutinous rice of this region(Olsen and Purugganan, 2002). Amylose content of rice endosperm is

92 therefore a major domestication trait thathas evolutionary importance. Among the minor alleles

93 affecting amylose content, a polymorphic $\mathrm{CT}_{\mathrm{n}}$ microsatellite in the $5^{\prime}$-untranslated region (UTR) of

94 Wx exon 1 has been shown to be correlated with various AC classes(Ayres et al., 1997). Other SNPs

95 in coding regions of different $W x$ alleles lead to decreased AC by dropping the binding efficiency of

96 GBSS-I to starch granules, changing amino acid at respective sites(Liu et al., 2009; Sato et al., 2002)

97 andaltering expression pattern of GBSS-I in endosperm and anthers(Mikami et al., 1999).In addition

98 to $W x$, mutations in genes such asDull(Zeng et al., 2007) and shrunken(Asaoka et al., 1993) also

99 indirectly affect AC in rice.

100

101 Bangladesh is the world's fourth largest rice producing country. It is considered as an enriched rice 102 germplasm reservoir with over 6500 wild, landraces and modern high yielding varieties (Elias et al., 103 2011).Majority of the rice species in Bangladeshare indica varieties with high amylosecontent 104 andnon-glutinous phenotype (Olsen and Purugganan, 2002). Several glutinous rice varieties, which 105 are locallyknown as 'Beruin'or sticky rice, are also cultivated in the Northeastern region of 106 Bangladesh(Dipti et al., 2003). These Beruin cultivars generally have low amylose content and are 107 popular for making traditional dishes on special occasions.However the molecular basis of low 108 amylose content in these local Beruin varieties is unknown. In this study we examined the nucleotide 109 diversity at the Wxlocusof selected Bangladeshi glutinous Beruin and non-glutinous rice cultivarsto 110 identify the underlying cause of $\mathrm{AC}$ variation in these genotypes. We also compared these

111 Bangladeshi varieties with known $W x$ haplotypes in an attempt to understand their evolutionary 112 pattern across Asia.

\section{2. Materials and Methods}

116 2.1.Plant materials

117 Seeds of eleven Beruin cultivars, which are representative of the diverse traditional landraces unique 118 to the Northeastern region of Bangladesh (Sylhet), were collected from the local farmers.An equal 
119 number of non-glutinous cultivars was then selectedto allow identification of SNPs underlying the

120 glutinous phenotype in Bangladeshi cultivars (Fig. 1A and 1B). Nine of the selected non-glutinous

121 cultivarswere from the same region as the Beruin cultivars. Two other popular aromatic

122 cultivars,Kalijira and Kataribhog, were collected fromthe Northwesternregion of Bangladesh

123 (Dinajpur) to expand the genetic diversity of sampled non-glutinous cultivars. All of the selected

124 cultivarsaretraditional transplanted Amanor monsoon season varieties. The collected seeds were

125 multiplied during the planting season in the net house.Three plants of each cultivar were grown ina

126 single pot to collect immature seeds.

128 2.2. Estimation of amylose content

129 The amylose content (AC) of all cultivars was determined according to previously

130 publishedprotocol(Juliano et al., 1981). Nazirshail rice flour (AC 25\%, Bangladesh Rice Research

131 Institute) was used as a control and potato starch flour (E.Merck, Germany) was used for generating

132 the standard curve.

133

134 2.3.Genomic DNA Extraction

135 Genomic DNA was extracted from leavesof 3-week-old pooled plants (10-12 plants percultivar)using

$136 \mathrm{CTAB}$ extractionmethod and in some cases by using the Qiagen DNeasy® plant mini kit. DNA of all

137 cultivars was quantified by spectrophotometry. Quality of extracted DNA was checked by $1 \%$ agarose

138 gel electrophoresis and compared with known concentration of Lambda DNA (Invitrogen, USA).

$140 \quad$ 2.4.PCR amplification and DNA sequencing

$141 \mathrm{~A} 2.7 \mathrm{~kb}$ upstream regulatory region of the Wxgene including promoter, exon 1, intron 1 and part of 142 exon 2 was PCR amplified and sequenced in all 22 Bangladeshi rice cultivars.PCR amplification was 143 performed in two steps to amplify overlapping $\sim 1.5 \mathrm{~kb}$ segments of the above upstream regulatory 144 region using primers described by Olsen and Purugganan (2002) (Supplementary Table 1). For each 145 primer set, $15 \mu \mathrm{l}$ PCR reaction mixture containing $60 \mathrm{ng}$ of DNA template, $1 \times$ PCR buffer, $1.67 \mathrm{mM}$ $146 \mathrm{MgCl}_{2}, 100 \mu \mathrm{M}$ of each dNTP, 0.33 pmol Forward /Reverse primer, 1 unit Taq DNA polymerase and 
1470.1 unit $\mathrm{Pfx}{ }^{\circledR}$ DNA polymerase (Invitrogen, USA) was used. Amplification conditions were $94{ }^{\circ} \mathrm{C}$ for

$1485 \mathrm{~min}$, followed by 35 cycles of $95{ }^{\circ} \mathrm{C}$ for $1 \mathrm{~min}, 65.2^{\circ} \mathrm{C}$ for $1 \mathrm{~min}, 72{ }^{\circ} \mathrm{C}$ for $1 \mathrm{~min}$ and a final 149 extension of $72{ }^{\circ} \mathrm{C}$ for $8.30 \mathrm{~min}$.

150

151 For further characterization of theWx coding region inShamudrophena, Kathali Beruin Red, Modhu

152 Beruin White, Khara Beruin and Mou Beruin,two different segments were amplified and sequenced

153 using four sets of primers (Supplementary Table 1).The first segment $(\sim 0.2 \mathrm{~kb})$ includedpart of exon

154 2, which is known to contain a 23 bp duplication in some glutinous varieties. This segment 155 wasamplified using primer pair sequences Glu-23F and Glu-23R asdescribed by Wanchana et al.

156 (2003).The other $\sim 2 \mathrm{~kb}$ segment including exons 3-10 was amplified using three overlapping primer

157 pairs (Supplementary Table 1). These primers were designed using primer3 program

158 (http://frodo.wi.mit.edu/primer3/) based on sequence of Nipponbare Wx locus(OS06G0133000)in

159 Gramene database (www.gramene.org). PCR amplification conditions were the same as for the $W x$

160 upstream regulatory region except the annealing temperature, whichwas optimized for each primer 161 pair (Supplementary Table 1).

162

163 All PCR amplicons were purified using QiAquick gel extraction kit (Qiagen, Germany) and directly 164 sequenced inABI 3730XL genetic analyzerusing both forward-reverse and internal primers 165 (Supplementary Table 1).Each fragment was sequencedtwice for SNP confirmation.All of the 166 sequences from this study have been deposited in GenBank (accession no.JF834042.1-JF834063.1

167 and KP675770-KP675774).

169 2.5.Sequence analysis

170 Wx upstream regulatory regionsequences from 22 Bangladeshi rice landraces were compared to 18 171 previously reported haplotypesacross Asia (Genbank accessions AY136760-AY136784). Allmultiple 172 sequence alignments were generated usingClustalW (Thompson et al., 2002).SNP/indels were 173 identified using TASSEL (Bradbury et al., 2007).Neighbour-joining treeof aligned Wx upstream 174 regulatory region $(2.7 \mathrm{~kb})$ sequenceswasconstructed usingMEGA 6.06 software package (Tamura et 
175 al., 2013) with Kimura two-parameter model and complete deletion of alignment gaps.Bootstrap test

176 with 1000 replicates was performedto ensure confidence in phylogeny. A median-joininghaplotype

177 network was also generated based on the aligned sequences using SplitsTree 4.13.1 (Huson and

178 Bryant, 2006). Motif change patterns due to SNPs in Wx promoter region and intron 1 of Bangladeshi

179 cultivars were predicted using the PLACE database (www.dna.affrc.go.jp/PLACE). Amino acid

180 changes due to SNP in sequenced $W x$ codingexons from selected Bangladeshi landraces were

181 predicted using the ExPASy translation tool (http://web.expasy.org/translate/).SplicePredictor

182 (http://bioservices.usd.edu/splicepredictor/) was used to predictsplice-site modifying SNPs in intronic

183 regions of sequenced varieties. RegRNA (http://regrna.mbc.nctu.edu.tw/) was used to predictcis-

184 regulatory elements involved in mRNA splicing or transcriptional regulation.

185

186 2.6.Semi-quantitative RT-PCR and gene expression

187 Total RNAsfrom rice endosperm at 16-18 days after floweringwere extracted using TRIZOL. First

188 strand cDNA were synthesized from total RNAfollowing manufacturer's protocol (Invitrogen,

189 USA).G3PDH (glyceraldehyde-3-phosphate dehydrogenase) gene-specific primers (Supplementary

190 Table 1) were used to optimize the cDNAconcentration for estimating the $W x$ mRNA level. Nanodrop

191 spectrophotometer was used to measure and optimize the working concentration of the

192 cDNA.WxmRNA level was assessed using cycle-dependent semi-quantitative RT-PCR with the

193 WxRT1 primer pair (Supplementary Table 1) for 30, 32 and 35 cycles.Expression levels of samples

194 wereestimated by visualinspection after electrophoresis in a $0.9 \%$ agarose gel based on the band

195 intensity of the loading control G3PDH. Two sets of primer pairs - Wx-RT2 and Wx-RT3

196 (Supplementary Table 1) were used to confirm the G/T SNP at the $5^{\prime}$-splice donor site of WxmRNA

197 of Bangladeshi cultivars.Wx-RT2 primer pair was used to determine the splicing efficiency of the first

198 intron in the selected landraces.Nipponbare was used as a controlas it contains G/T SNP at intron1

199 splice donor site of $W x$ gene (Liu et al., 2009).

200

201

3. Results

202 
204 In order to identify the molecular basis of glutinous rice in Bangladesh, we first evaluated the amylose 205 content (AC) of22Beruin and non-Beruintraditional landracesthat arelocally considered as glutinous 206 and non-glutinous cultivars respectively. These selected cultivars were sampled fromintensive rice 207 cultivation zones in Northeastern and Northwestern regions of Bangladesh that are known to harbour 208 many diverse landraces(Elias et al., 2011). Based on their AC, the selected cultivars were assigned 209 intothree major categories - intermediate, low and glutinous/waxy. All non-BeruinBangladeshi 210 cultivars in this study had intermediate AC ranging from $19.1 \%$ to $23.3 \%$ (Fig.1A), confirming that

211 these are indeed non-glutinous. In contrast, only sevenout of elevenBeruin varieties, traditionally 212 associated as glutinous or sticky rice, had amylose content within the waxy range (0-5.4\%), as shown

213 in Fig. 1B.Among the remaining four Beruin varieties, Kathali Beruin Red and Kalo Beruin belonged 214 to the low AC group (AC 13.7\% and 8.2\% respectively). The two other cultivars Modhu Beruin Red 215 and Push Beruin (AC 23.4\% and 22.6\% respectively) had intermediate AC, similar to the Bangladeshi 216 non-glutinous cultivars.

218 3.2.Nucleotide variation at Wxupstream regulatory region of Bangladeshicultivars

219 We compared the $\sim 2.7 \mathrm{~kb} W x$ upstream noncoding regionsequencesfromselectedBangladeshi 220 cultivarsto Olsen and Purugganan (2002) reported progenitor haplotypes F (non-glutinous) and G 221 (glutinous). Theseprogenitor haplotypes differ only in the intron1 G/T splice donor site mutation(Olsen and Purugganan, 2002).Overall, Bangladeshi cultivarshad 29 single nucleotide polymorphisms (SNP) and 11 insertions/deletions (indel) in the sequencedupstream regulatory region

224 (Fig.1C). Out of these, 15SNP and 3 indelswere found in the promoterregions and 13 SNPand 7indels 225 in intron 1. TheseSNP and indels in promoter and intron 1 were more frequent in non-glutinous 226 cultivarsthan the Beruin cultivars. In total, seven different microsatellite alleles: $\mathrm{CT}_{8}, \mathrm{CT}_{10}, \mathrm{CT}_{11}$, $227 \mathrm{CT}_{12}, \mathrm{CT}_{16}, \mathrm{CT}_{17}$ and $\mathrm{CT}_{18}$, were identified in the $W x$ exon 1 (encoding $5^{\prime}$ untranslated region) of 228 Bangladeshi cultivars(Figure 1C). An additional SNP withinWx exon 2 that also codes for 5' UTR was 229 observed only in one of the non-glutinous Bangladeshi cultivars. 
230 All of the Bangladeshi non-glutinous cultivars in this study had G-SNP at the $5^{\prime}$ splice donor site

231 ofWxintron 1 (Fig.1C)similar to the known non-glutinous haplotype $\mathrm{F}$. The $\mathrm{CT}_{10}$ or $\mathrm{CT}_{11}$ alleles within

$232 W x$ exon 1 were observed in six of these non-glutinous Bangladeshi cultivars. $\mathrm{TheCT}_{17}$ allele was

233 present in cultivars with variable AC, ranging from intermediate AC in Bangladeshi non-glutinous

234 cultivars to low and waxy $\mathrm{AC}$ content in the Beruin cultivars (Fig. 1). The $\mathrm{CT}_{12} / \mathrm{CT}_{18}$ and $\mathrm{CT}_{8} / \mathrm{CT}_{16}$

235 alleles werespecific to non-glutinous and glutinous Bangladeshi cultivars, respectively.

237 G/T SNP at the 5' splice site of $W x$ intron 1 could explain the waxy or low AC phenotype (AC 4.4238 8.2\%) of six out of eleven Bangladeshi Beruin cultivars. These cultivars also carried $\mathrm{CT}_{17}$ allele in 239 exon 1 similar to theknown glutinous progenitor haplotype G (Fig. 1C). Among these six Beruin 240 cultivars, Pak Beruin, Kathali Beruin white and Kalo Beruin had an additional novel G/A SNP at 241 position 718 in the $W x$ promoter region (Fig. 1C). The remaining fiveBeruin cultivars lackedthe G/T

242 SNP in intron 1 splice donor site. Among these, Push Beruin and Modhu Beruin Red also showed 243 intermediate amylose phenotype (AC 22.6\% and $\mathrm{AC} 23.4 \%$ respectively) like non244 glutinousBangladeshi cultivars in this study(Fig. 1A). These varieties also had other nucleotide variations in $W x$ regulatory region that were similar to the Bangladeshi non-glutinous cultivars (Fig.

246 1C). This confirmed that Push Beruin and Modhu Beruin Red might be locally misnamed as glutinous 247 varieties even though they have non-glutinous phenotype.

249 The other three Beruin cultivars that lacked the G/T SNP in splice donor site(Khara Beruin, Modhu 250 Beruin Whiteand Kathali Beruin Red) had waxy or low AC phenotype (Fig. 1B). Among these only 251 Khara Beruin showed several unique mutationsin the Wx upstream noncoding region (T/A SNP at 216 252 position in promoter, G/T SNP at position 1934 in intron 1).This indicated that polymorphisms other 253 than G/T SNP in intron 1 splice donor site and $\mathrm{CT}_{\mathrm{n}}$ allele in exon 1 may play a major role in 254 regulating $W x$ gene expression in these three genotypes.

3.3.Evolutionary relatedness of Bangladeshi cultivars

257 An unrooted phylogenetic tree based on the aligned $\sim 2.7 \mathrm{~kb} W x$ upstream regulatory region sequences 
of selectedBangladeshi cultivars and 18 other reported haplotypes (Olsen and Purugganan, 2002)was constructed to explore the evolutionary relationships among theseaccessions. These 18 reported

260 haplotypes representthe landrace diversity across Asia. In the phylogenetic tree,six Bangladeshi 261 glutinous cultivars (Akia Beruin White, Akia Beruin Red, Mou Beruin, Kalo Beruin, Kathali Beruin 262 White and Pak Beruin)clustered with reported glutinous haplotypes G, I, K, L and Q at high bootstrap 263 (98\%) value (Fig.2). Pak Beruin, Kalo Beruin, Kathali Beruin White along with a non-glutinous 264 cultivar Shamudrophena also formed a sub clade depending on theG/A SNP in position 718 of the Wx 265 promoter region (Fig.2). These cultivars may carry the same $W x$ allele or with an additional mutation $266(718 \mathrm{G} / \mathrm{A})$.

On the other hand, five glutinous Beruin cultivars that lacked the intron 1 G/T SNP were grouped 269 with reported non-glutinous haplotypes and Bangladeshi non-glutinous cultivars. Intermediate 270 ACcultivars Push Beruin and Modhu Beruin Red clustered with reported non-glutinous haplotypes B, 271 Aand Bangladeshi non-glutinous cultivars (Fig.2).Waxycultivar Khara Beruin clustered with reported non-glutinous haplotypes $\mathrm{E}$ and $\mathrm{D}$ at $64 \%$ bootstrap and with non-glutinous haplotype $\mathrm{C}$ at $93 \%$

273 bootstrap (Fig.2).Based on the aligned $W x$ upstream regulatory region sequences, waxy AC Modhu

274 Beruin White and low AC Kathali Beruin Red appeared to be very close to intragenic recombinant 275 haplotype R andnon-glutinous haplotype S, respectively, even thoughneithershared similaramylose 276 phenotype with the corresponding haplotypes(Fig.2).Bangladeshi non-glutinouscultivars were 277 clustered with reported non-glutinous haplotypes but were more close to the haplotype F, A and B 278 (Fig.2).In addition, Chinigura, Kalijira and Kataribhog distinctly clustered withnon-glutinous 279 progenitor haplotype $\mathrm{F}$ at $48 \%$ bootstrap (Fig.2). Itmight be possible that the Bangladeshi non280 glutinouscultivarsoriginally carried the non-glutinous progenitor Waxyallele and then evolved into 281 haplotypes A and Bthrough subsequent mutations at promoter region and intron 1.

283 3.4.Nucleotide variationsat coding regionof $W x$ gene inselected Beruin cultivars

284 Since G/T SNP at intron 1 splice donor site was not enough to explain the waxy AC of Modhu Beruin 285 White and Khara Beruin and low AC of Kathali Beruin Red, we further sequenced two segments in 
286 thecoding region of $W x$ gene. Mou Beruin (AC 4.4\%) and Shamudrophena (AC 23.4\%) were

287 consideredas references for Bangladeshi glutinous andnon-glutinouscultivars respectively for this 288 analysis. The first sequenced segment in $W x$ gene included part of coding region of exon $2(\sim 196 \mathrm{bp})$ 289 which has been reported to contain a 23bp frame-shift duplication in some glutinous varieties that lead 290 to non-functional Wx proteins (Mikami et al., 2008; Wanchana et al., 2003). Among the selected 291 cultivars, this 23bp duplication was only identified in Mou Beruin, consistent with its glutinous 292 phenotype (Table 1).The second segment analyzed in $W x$ gene spanned $\sim 1.9 \mathrm{~kb}$ genomic region 293 including 3' end of exons 2-10 and introns 2-10.Only the non-glutinous reference Shamudrophena 294 among the five cultivars carried A/C SNP in exon 6 (position 671 from start codon), which resulted in 295 nonsynonymous amino acid change from tyrosine to serine (Table 1). This SNP has been shown to be 296 associated with intermediate AC (21-24\%) previously(Dobo et al., 2010). Two other coding region $297 \mathrm{SNPs}(\mathrm{T} / \mathrm{C}$ in exon 9,C/T in exon 10) were identified inthe three selected Beruin cultivars but not in 298 the non-glutinous or glutinous reference (Table 1). Exon9 SNP (T/C, position 1109 from start codon) 299 was found in all three selected cultivars (Table1). The exon9 SNPresults in a synonymouschange 300 inamino acid and has been previously reported as a silent mutation(Larkin and Park, 2003). Exon 10

301 SNP $(\mathrm{C} / \mathrm{T})$ was found only in low AC Kathali Beruin Red (Table 1). Exon 10 SNP (C/T) caused a 302 nonsynonymous change from a nonpolar amino acid proline (CCT) to polar amino acid Serine (TCT), 303 and it has been reported that this SNP is linked to high amylose content(Larkin and Park, 2003).

304 Therefore these exon-specific mutations were not directly responsible for the variant AC phenotype 305 traits observed for the selectedBeruin cultivars.

307 Most interestingly, Kathali Beruin Red, Modhu Beruin White and Mou Beruin had overall four SNPs 308 and two indels in $W x$ introns which wereabsentin the Bangladeshi non-glutinous and glutinous 309 references (Table 1). Among these variations, $\mathrm{C}$ deletion in intron 5, G/A (at positions 81 and 95 from 3105 ' splice site of intron 10) and ATA deletion at position 104-106in intron 10 were identified in all 311 these three cultivars.On the other hand, G/A in intron 10 (position 118) was found only in low AC 312 Kathali Beruin Red and A/G at position 29 from 5' splice site of intron 9was only observed in the 313 waxy ACKhara Beruin and Modhu Beruin White.A search for putative cis-elements that might be 
314 affected by these SNPs/indel revealed that the $\mathrm{C}$ indel was located within a poly-C tract in intron 5,

315 and this indel resulted in absence of a putative INTRONLOWER motif in these three cultivars

316 (Supplementary Table 2). Also, the G/A indel in intron 10 (at position 95 from 5' splice site) resulted

317 in a missing putative ESE-SF2/ASF motif (Exonic Splicing Enhancer) in these three cultivars 318 (Supplementary Table 2).

320 Further homology search using NCBI BLASTn revealedthat the sequenced coding regions of Khara 321 Beruin and Modhu Beruin White shared $\sim 99 \%$ identity with previously reported $W x^{o p}$ (opaque) allele containing cultivars ARC10818, ACC35618 and ARC6622 (Genbank accessions AB281448.1, 323 AB281447.1, and AB281453.1) from Nepal and India (Mikami et al., 2008, 1999). These $W x^{o p}$ 324 accessions did not contain the G/T SNP at the 5' splice donor site of intron 1 and also had 325 nonsynonymous A/G SNP in exon 4 at position 521 from start codon (Mikami et al., 2008, 1999).

326 However the latter exon 4 SNP was not present in the Khara Beruin and Modhu Berun White (Table 327 1). Comparison of the rice grains revealed that only Khara Beruin had a completely opaque or chalky 328 endosperm, while Modhu Beruin White had partially chalky endosperm (Fig. 3), which supports the 329 low to very low amylose content of these varieties.

331 3.5.Wx gene expression in selected Beruin cultivars without G/T SNP in splice donor site

332 Semi-quantitative RT-PCR was used to estimate total expression levels of Wx gene in Modhu Beruin 333 White (waxy AC), Kathali Beruin Red(low AC), Push Beruin and Modhu Beruin Red (intermediate 334 AC). Nipponbarewas used as a controlsince it is known to show decreased splicing efficiency of $W x$ 335 transcript due to G/T SNP at intron 1 splice donor site (Liu et al., 2009). The results showed that $W x$ 336 transcript levelsin Push Beruin, Modhu Beruin Red andKathali Beruin Red were relatively much 337 higher compared to the control Nipponbare, (Fig. 4A). This suggests that the splicing efficiency of $W x$ 338 transcript is not affected in Push Beruin, Modhu Beruin Red as well as Kathali Beruin Red. This 339 observation is consistent with the absence of G/T SNP at intron 1 and the intermediate-to-low AC in 340 these cultivars. The expression level of $W x$ transcript in Modhu Beruin White (intron 1 splice site 341 mutation absent)was also higher than that of Nipponbare but lower than the aforementioned three 
342 Beruin cultivars (Fig.4A).The transcript level however correlates with the waxy AC of Modhu Beruin 343 White.

344 RT-PCR with primers specific for unspliced variant of $W x$ transcript (Liu et al., 2009) confirmedthe 345 absence of intron 1 containing $1.1 \mathrm{~kb}$ fragment in all of the selected Beruincultivars(Fig. 4B).A 346 second set of RT-PCR primers previously described by Prathepha (2007) was used to re-confirm the 347 absence of G/T SNP at intron1 splice donor site of $W x$ gene of these selected cultivars (Fig. 348 4C).Nipponbare showed a predominant band at $120 \mathrm{bp}$ and another band at $210 \mathrm{bp}$, while the selected 349 Beruin cultivars only showed the 210 bp band (Fig. 4C). This is consistent with previous observations thatcultivars carrying intron $1 \mathrm{G} / \mathrm{T}$ SNP showeda predominant band at $120 \mathrm{bp}$ with an additional band

351 at $210 \mathrm{bp}$, indicating at least two alternative variants of $W x$ transcript,but cultivars without the 352 mutation showed only a predominant band at 210bp(Prathepha, 2007).

\section{4.Discussion}

4.1.Distribution of Bangladeshi rice cultivars in context with Asian rice cultivars

357 In this study, 22 Bangladeshi glutinous and non-glutinous cultivars were placed in specific positions

358 along with 18previously reportedWx haplotypes(Olsen and Purugganan, 2002)based on phylogenetic359 and haplotype network analysesofWx upstream regulatory region sequences (Fig.2 and 5).The

360 previously reported Wxhaplotypes were identified using 105 accessionsfrom different countriesof 361 Asia(Olsen and Purugganan, 2002).Bangladeshi non-glutinous cultivars Kalijira, Kataribhog and 362 Chinigura have the same $W x$ allele as haplotype $\mathrm{F}$ with no additional mutation.As such, these cultivars

363 wereplaced with non-glutinous progenitor haplotype F. Previously, Olsen and Purugganan (2002) also 364 showed that $39 \%$ of accessions in Southeast Asia and $15 \%$ of the South Asian accessions contain this 365 F haplotype. Four non-glutinous cultivars (Lathial White, Lathial Red, Girishail, and Raujan2) and 366 one intermediate amylose containing cultivar Modhu Beruin Red bear closer identity to haplotype A 367 and were placed with it (Fig.5). 
369 Three other Bangladeshi non-glutinous cultivars - Balam White, Balam Red and Raujan, and one

370 intermediate amylose containing cultivar Push Beruin were placed in the same node as haplotype B

371 (Fig.5). Non-glutinous haplotypes A and B were derived from haplotype F by subsequent mutations at

372 both promoter and intron1 region. Haplotype A is more frequent in Southeast Asia and haplotype B is

373 more frequent in South Asia(Olsen and Purugganan, 2002). Therefore, Bangladeshinon-glutinous and

374 Beruin rice cultivars with intermediate AC carry the general pattern prevalent in South and Southeast

375 Asia.

376

377 Among the Bangladeshi glutinous cultivars, Mou Beruin, Akia Beruin White and Akia Beruin Red

378 have the same sequence identities as the glutinous progenitor haplotype $\mathrm{G}$ in $W x$ upstream region

379 (Fig.5). Most of the glutinous rice accessions in Southeast, North and South Asia have been reported

380 to carry haplotype G. Bangladeshi glutinous cultivarsKalo Beruin, Pak Beruin and Kathali Beruin

381 White containedan additional G/ASNP at position 718of Wx promoter along with the G/T SNP at the

$3825^{\prime}$ splice donor site of intron 1 (Fig. 1).These cultivars clustered with the glutinous haplotype group

383 (L, K, O and I), whichalso have additional mutations located at promoter and intron 1 but at different

384 positions (Fig.5). The 718 (G/A) SNP has not yet been reported and is therefore unique to

385 Bangladeshi glutinous cultivars as well as the non-glutinous Shamudrophena.

387 From the median-joining network analysis(Fig.5), low AC Bangladeshi Kathali Beruin Red was found 388 to be close to the non-glutinous haplotype S.Two other waxy AC cultivars Khara Beruin and Modhu 389 Beruin White were placed with non-glutinous haplotype $\mathrm{C}$ and recombinant haplotype $\mathrm{R}$ in theWx 390 haplotype network (Fig.5). This suggests that some Bangladeshi glutinous Beruin cultivars may have 391 a different allele compared to glutinous cultivars of Asia responsible for waxy or low AC phenotype.

4.2. Bangladesh genotype-specific changes

394 Several studies have reported specificSNP or indelsin Wxexonsthat decrease AC in rice seeds by

395 reducing the binding of GBSS-I to starch granules or creating stop codon that terminates the 396 translation of $W x$ transcript (Liu et al., 2009; Wanchana et al., 2003). However, waxy Khara Beruin, 
397 Modhu Beruin White and Kathali Beruin did not have any of those SNPs.Pairwise sequence

398 comparisons of these cultivars with Olsen and Purugganan (2002) reported haplotypesshowed that 399 some mutations at both promoter and intron 1 region were shared between Khara Beruin and 400 haplotype C (99.93\% sequence identity), Modhu Beruin White and haplotype R (99.19\% identity) as 401 well as Kathali Beruin Red and haplotype S (99.26\% identity). Some of these mutations were found to 402 be located within putative cis-regulatory motifs in the $W x$ promoter site in Beruin cultivars 403 (Supplementary Table 2). Most interestingly, these three cultivars have a $\mathrm{C}$ deletion at a 404 polypyrimidine tract of intron 5 as well as twoG/A SNPs and an ATA deletion at intron 10. This C 405 deletion in intron 5 consequentially causesloss of theputativeINTRONLOWER motif in that 406 region.This may causeslippage of intron splicing and subsequent translation(Brown, 1986; Sridharan 407 and Singh, 2007). Also, the G/A indel in intron 10(at position 95 from 5' splice site) which affected a 408 putativeExonic Splicing Enhancer motif might cause exon skippingin these three cultivars 409 (Supplementary Table 2). Therefore, these mutations might be another cause of the lowAC in Khara 410 Beruin, Modhu Beruin White and Kathali Beruin Red despite lack of the G/T SNP at the intron 1 411 splice donor site, which is known as the main cause of waxy or low amylose phenotype.

413 Waxy amylose containing Khara Beruin and Modhu Beruin White also contained one SNP at intron 9 414 (G/A) and showed 99\% similarity with opaque or chalky endosperm containing rice cultivars found in 415 Nepal, India, Myanmar and Indonesia. On the other hand, low amylose containing Kathali Beruin Red 416 contained one G/A SNP in position 118 in intron10 that might create an alternative splice acceptor 417 site in intron 10 (Supplementary Table 2). This SNP in intron10 has not been reported previously. 418 These SNPs at introns 9 and 10 also might have an effect on splicing as well as gene expression and 419 varying amylose between waxy Khara Beruin, Modhu Beruin and Low AC Kathali Beruin 420 Red.However, the transcript levels of the $W x$ gene in these three Beruin cultivars were not low 421 compared to Nipponbare, whichcarries the G/T splice site SNP in intron 1. Whether the above SNPs 422 in the promoter and introns alone are responsible for regulating $W x$ transcript levels in these cultivars 423 or other players are involved requires further investigation. 
426 Bangladeshi non-glutinous and glutinous cultivars with intermediate AC carried the general pattern of nucleotide variation in $W x$ upstream region as observed in South and Southeast Asian cultivars. Some exceptional Wxalleles were also identified in Bangladeshi glutinous cultivars Khara Beruin, Modhu Beruin White and Kathali Beruin Red. These cultivars lack the G/T splice site mutationin intron1 regionas non-glutinous cultivars, but yet have waxy and/or low amylose phenotype. However these cultivarshave some SNPsin promoter region that may alterputative cis-regulatory motifsinvolved inWx gene regulation.Additionally, aputative INTRONLOWER motif (consensus sequence for plant introns) and an Exonic Splicing Enhancer motif were lost in these cultivars due to indelsin $W x$ introns5 and 10 respectively.Khara Beruin and Modhu Beruin White (waxy AC) also contained a SNP at intron 9 and Kathali Beruin Red (low AC) contained two SNPs at introns 6 and 10. It can be hypothesized that the intron- and promoter-specific mutations found in these three cultivars could be the cause of the waxy or low amylose phenotype. This hypothesis however needs to be investigated further throughfunctional characterization of these motifs and confirmation of alternative $W x$ variants due to misregulation of splicing.

\section{Acknowledgement}

442 Funding for this research, including fellowships for RB and SS and consumables and equipment,was received from the Bangladesh Chapter of USDA under the 416-(B) grant aid. BAS-USDA-PALS project also provided a 6-month extension fellowship to RB. We would like to thank Dr. Abdul Chaudhury and Munir Hasan for providing information on the Beruin cultivars of Bangladesh and encouraging us to undertake the work, Md. Sazzadur Rahman (Senior Scientific Officer, Bangladesh Rice Research Institute) for helping with seed collection and Md. Shamim Hossain for taking care of the plants.

References

451 Asaoka, M., Okuno, K., Yano, M., Fuwa, H., 1993. Effects of Shrunken and Other Mutations on the Properties of Rice Endosperm Starch. Starch/Stärke 45, 383-387. 
Ayres, N.M., McClung, A.M., Larkin, P.D., Bligh, H.F.J., Jones, C.A., Park, W.D., 1997. Microsatellites and a single-nucleotide polymorphism differentiate apparentamylose classes in an extended pedigree of US rice germ plasm. Theoretical and Applied Genetics 94, 773781.

Bergman, C., Bhattacharya, K., Ohtsubo, K., 2004. Rice end-use quality analysis. In: Champagne,

Bradbury, P.J., Zhang, Z., Kroon, D.E., Casstevens, T.M., Ramdoss, Y., Buckler, E.S., 2007. TASSEL: software for association mapping of complex traits in diverse samples. Bioinformatics 23, 2633-2635.

Brown, J.W.S., 1986. A catalogue of splice junction and putative branch point sequences from plant introns. Nucleic Acids Research 14, 9549-9559.

Cai, X.L., Wang, Z.Y., Xing, Y.Y., Zhang, J.L., Hong, M.M., 1998. Aberrant splicing of intron 1 leads to the heterogeneous $5^{\prime}$ UTR and decreased expression of waxy gene in rice cultivars of intermediate amylose content. The Plant Journal 14, 459-465.

Dipti, S., Bari, M., Kabir, K., 2003. Grain quality characteristics of some Beruin rice varieties of

Dobo, M., Ayres, N., Walker, G., Park, W.D., 2010. Polymorphism in the GBSS gene affects amylose content in US and European rice germplasm. Journal ofCereal Science 52, 450-456.

Dong, B., 2000. The potential commercial value of Yunnan "Zhefang rice." Yunnan Agric. Sci. Technol. 5, 13-15.

Elias, S.M., Hasan, A.K.M.M., Seraj, Z.I., 2011. Microsatellite marker diversity and sequence polymorphism in the red gene locus of indigenous rice populations of Bangladesh. Plant Systematics and Evolution 296, 157-165.

Fan, C.C., Yu, X.Q., Xing, Y.Z., Xu, C.G., Luo, L.J., Zhang, Q., 2005. The main effects, epistatic effects and environmental interactions of QTLs on the cooking and eating quality of rice in a doubled-haploid line population. Theoretical and Applied Genetics 110, 1445-1452.

Huson, D.H., Bryant, D., 2006. Application of Phylogenetic Networks in Evolutionary Studies. Molecular Biology and Evolution. 23, 254-267. 
Inukai, T., Sako, A., Hirano, H.Y., Sano, Y., 2000. Analysis of intragenic recombination at wx in rice: Correlation between the molecular and genetic maps within the locus. Genome 43, 589-596.

Juliano, B.O., Perez, C.M., Blakeney, A.B., Castillo, T., Kongseree, N., Laignelet, B., Lapis, E.T., Murty, V.V.S., Paule, C.M., Webb, B.D., 1981. International Cooperative Testing on the Amylose Content of Milled Rice. Starch/Stärke 33, 157-162.

Larkin, P.D., Park, W.D., 2003. Association of waxy gene single nucleotide polymorphisms with starch characteristics in rice (Oryza sativa L.). Molecular Breeding 12, 335-339.

Liu, L., Ma, X., Liu, S., Zhu, C., Jiang, L., Wang, Y., Shen, Y., Ren, Y., Dong, H., Chen, L., others, 2009. Identification and characterization of a novel Waxy allele from a Yunnan rice landrace. Plant Molecular Biology 71, 609-626.

Martin, C., Smith, A.M., 1995. Starch biosynthesis. Plant Cell 7, 971-985.

Mikami, I., Aikawa, M., Hirano, H.Y., Sano, Y., 1999. Altered tissue-specific expression at the Wx gene of the opaque mutants in rice. Euphytica 105, 91-97.

Mikami, I., Uwatoko, N., Ikeda, Y., Yamaguchi, J., Hirano, H., Suzuki, Y., Sano, Y., 2008. Allelic diversification at the $w x$ locus in landraces of Asian rice. Theoretical and Applied Genetics $116,979-989$.

Morishima, H., Sano, Y., Oka, H.I., 1992. Evolutionary studies in cultivated rice and its wild relatives. Oxford Surveys in Evolutionary Biology 8, 135-135.

Okagaki, R.J., Wessler, S.R., 1988. Comparison of non-mutant and mutant waxy genes in rice and maize. Genetics 120, 1137-1143.

Olsen, K.M., Purugganan, M.D., 2002. Molecular evidence on the origin and evolution of glutinous rice. Genetics 162, 941-950.

Prathepha, P., 2007. Identification of variant transcripts of waxy gene in non-glutinous rice (O. sativa L.) with different amylose content. Pakistan Journal of Biological Sciences 10, 2500-2504.

Sato, H., Suzuki, Y., Sakai, M., Imbe, T., 2002. Molecular Characterization of Wx-mq, a Novel Mutant Gene for Low-amylose Content in Endosperm of Rice (Oryza sativa L.). Breeding Science 52, 131-135.

Sridharan, V., Singh, R., 2007. A conditional role of U2AF in splicing of introns with unconventional 
polypyrimidine tracts. Molecular and Cellular Biology 27, 7334-7344.

510 Tamura, K., Stecher, G., Peterson, D., Filipski, A., Kumar, S., 2013. MEGA6: molecular evolutionary

511 genetics analysis version 6.0. Molecular Biology and Evolution 30, 2725-2729.

512 Thompson, J.D., Gibson, T.J., Higgins, D.G., 2002. Multiple Sequence Alignment Using ClustalW

513 and ClustalX. In: Baxevanis, A.D., Stein, L.D., Stormo, G.D., Yates, J.R. (Eds.), Current

514 Protocols in Bioinformatics. John Wiley \& Sons, New York, pp. 2.3.1-2.3.22.

515 Wanchana, S., Toojinda, T., Tragoonrung, S., Vanavichit, A., 2003. Duplicated coding sequence in 516 the waxy allele of tropical glutinous rice (Oryza sativa L.). Plant Science 165, 1193-1199.

517 Yamanaka, S., Nakamura, I., Watanabe, K.N., Sato, Y.I., 2004. Identification of SNPs in the waxy 518 gene among glutinous rice cultivars and their evolutionary significance during the 519 domestication process of rice. Theoreticaland Applied Genetics 108, 1200-1204.

520 Zeng, D., Yan, M., Wang, Y., Liu, X., Qian, Q., Li, J., 2007. Du1, encoding a novel Prp1 protein, 521 regulates starch biosynthesis through affecting the splicing of $W x^{b}$ pre-mRNAs in rice (Oryza 522 sativa L.). Plant Molecular Biology 65, 501-509.

523 